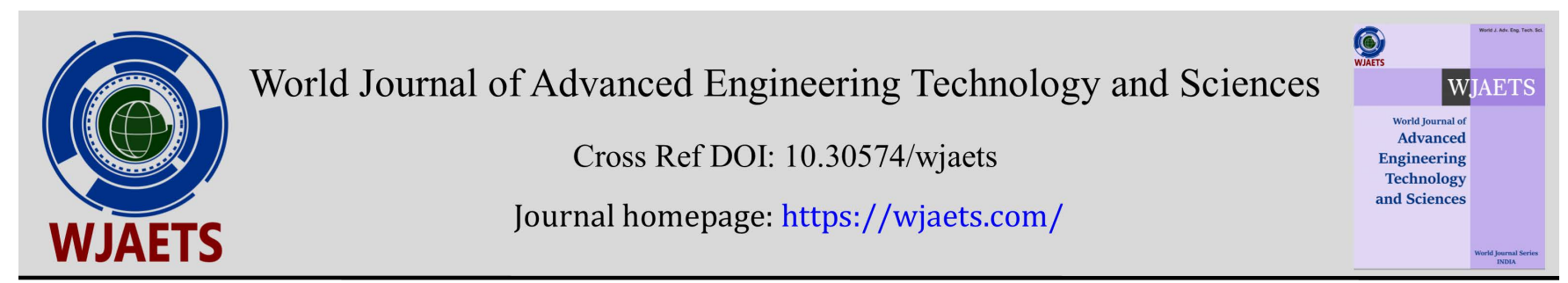

(RESEARCh ARTicle)

Check for updates

\title{
The rigid kinetic energy (RKE) concept for deflective suspended mobile robot in energy workspace
}

\section{Uchenna Christopher Akwara *}

School of Science and Engineering, PhD in Mechanical Engineering, Atlantic International University, Honolulu, USA.

World Journal of Advanced Engineering Technology and Sciences, 2021, 02(01), 001-010

Publication history: Received on 18 December 2020; revised on 29 December 2020; accepted on 02 January 2021

Article DOI: https://doi.org/10.30574/wjaets.2021.2.1.0019

\begin{abstract}
In this paper, the research study on the rigid kinetic energy (RKE) concept for deflective suspended mobile robot in energy workspace is adopted. This method is applied to solve and address the rigid kinetic problem, to specify the significant of the deflective coefficient problem, to derive a mathematical formation of rigid kinetic energy, to determine the nature of energy environment and to develop an algorithm to drive the rigid mobile robot in the energy workspace. The experiment was conducted on Lenovo Laptop with Microsoft window 10 operating system and MOBOT software application.
\end{abstract}

Keywords: Coefficient; Deflective; Rigid, Mobile robot; Kinetic energy; Actual; Virtual; Suspended; Workspace

\section{Introduction}

Within the context, rigid mobile robot in a deflective state, have the tendency of deflection in an insignificant coefficient eye point of the rigid mobile robot in a closed kinetic energy systems. As the deflection is optimum, the rigid mobile robot remains on a pivoted edge point to attain a rigid kinetic energy on the rigid motion. It takes an absolute application of the suspended rigid mobile robot to ascertain a maximum placement or drift from one dynamic state to another.

\section{Related work}

This section highlighted the previous related work carried out as a literature update which includes; Rigid body dynamics for rotational \& translation about a fixed axis [1]. In his research, he analyzed the motion of systems of particles and also of rigid bodies that are simply undergoing translational and rotational motion about a fixed direction of motion.

Revolutionary motion of a body under the action of resolution of acceleration due to gravity involved [2]. He established that the directional change in velocity of body revolving around a circular orbit can be understood depending on the basis of force gravitation appearing during the act of motion which shows in 2D effect mechanism.

Non-convex rigid bodies with stacking [3]. In their research, they proposed a novel approach to time integration merging it with the collision course and contact processing algorithms principle to a fashion that would obviates the need for ad hoc threshold velocity.

Rigid body motion [4]. He described how to use and implement the impulse force by keeping an object in a rest position and his methods assumed a very simple system of rigid bodies.

\footnotetext{
* Corresponding author: U.C. Akwara; Phone: +2348082169797; Email:uchenacha@yahoo.com

School of Science, PhD in Mechanical Engineering, Atlantic International University, Honolulu, USA.
} 
Study on the application of rigid body dynamics in the traffic accident reconstruction [5]. He established that the reconstruction of the vehicle collision accident purpose started from the final position of the vehicle and also builds the back projections by using the rigid body kinetic and rigid body model.

Interactive Manipulation of Rigid Body Simulation [6]. In their research, they described a novel interactive technique for intuitive manipulation of rigid multi-body simulations and the animation can select bodies at any time and drag them to desired locations.

Physics chapter 11-Rotational motion (The dynamics of rigid body) [7]. In their research papers, they established that a rigid body rotates with uniform angular speed $w$ about a fixed axis possesses kinetic energy of relevant rotation.

Computing the physical parameters of rigid-body motion from video [8]. He developed an algorithm that would take a video clip of a tumbling rigid body of known shape and generates a physical simulation of the object observed in the video clip.

A 3D rigid body dynamics for kinetic energy, instability and equations of motion [9]. In their research, they described a motion in term of given out a rotation about fixed mass and to solve the force system required to sustain the motion.

Within the research, this section identify and specify the problems associated with the rigid kinetic energy experimental research and provide some problem statements relating to rigid kinetic energy concept.

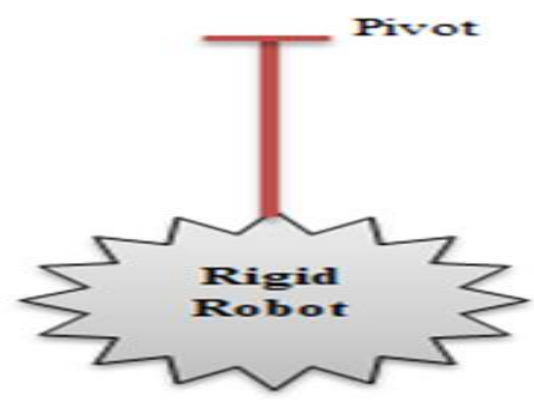

Figure 1 The sketch diagram of rigid mobile robot on a pivoted edge point.

The problem statements found on the (RKE) application during the research experiment includes;

- $\quad$ [1]. How does a rigid mobile robot be suspended on an edge point?

- [2].Is there any significant on the deflective coefficient of the rigid mobile robot?

- [3].Is there any rigid energy placement due to deflective motion involved?

- [4].What nature of environment is required for the energy workspace?

- [5].Is there any mathematical formula involved?

- [6].Is there any algorithm developed to drive the rigid mobile robot?

- [7].What mobile robot software is required for the (RKE) experience?

\section{Methodology}

This section highlighted the proposed method applied to solve the lingering problems acquired by the rigid mobile robot to gain rigid kinetic energy for deflective coefficient in energy workspace.

\subsection{Definition of Abbreviated Terms}

This section explained the definition of abbreviated terms used during the research and what it's stand for.

i. RKE: It stands for the rigid kinetic energy of deflective coefficient of rigid mobile robot in energy workspace. It is the product of rigid mass and the rigid velocity of deflective coefficient.

ii. V: It stands for the rigid velocity of deflective coefficient. It is the rigid mobile robot distance deflected with rigid time interval.

iii. $\quad$ t: It stands for the thickness of the rigid mobile robot. It is the physical nature of the rigid width to the base distance of the rigid deflective coefficient. 
iv. L: It stands for the length of deflection at the Centre. It is the cross-sectional distance of the deflective suspended rigid mobile robot.

v. K: It stands for the constant coefficient. It is the actual deflected value to the virtual deflected value of the rigid mobile robot.

vi. $\quad \mathbf{y}$ : It stands for the deflection at the center. It is the position of the virtual deflected value suspended at the edge point to the rigid mobile robot placement.

vii. $\quad$ n: It stands for the coefficient of rigid mobile robot. It is the calculated coefficient product of the overall length and the rigid diameter.

viii. M: It stands for the rigid mass of the mobile robot. It is the total mass acquired by the rigid mobile robot in the closed system.

ix. d: It stands for the rigid diameter of the mobile robot. It is the circumference diameter of the deflective coefficient.

\subsection{The Nature of Environment}

This section explained the nature of the environment for the rigid mobile robot. The rigid mobile robot remains in the energy workspace grid of the deflective coefficient. The application of mathematical algorithm to drive the rigid mobile robot in energy workspace makes the rigid mobile robot to experience Partially Known Environment (PKE).

\subsection{The Rigid Kinetic Energy (RKE) Algorithm}

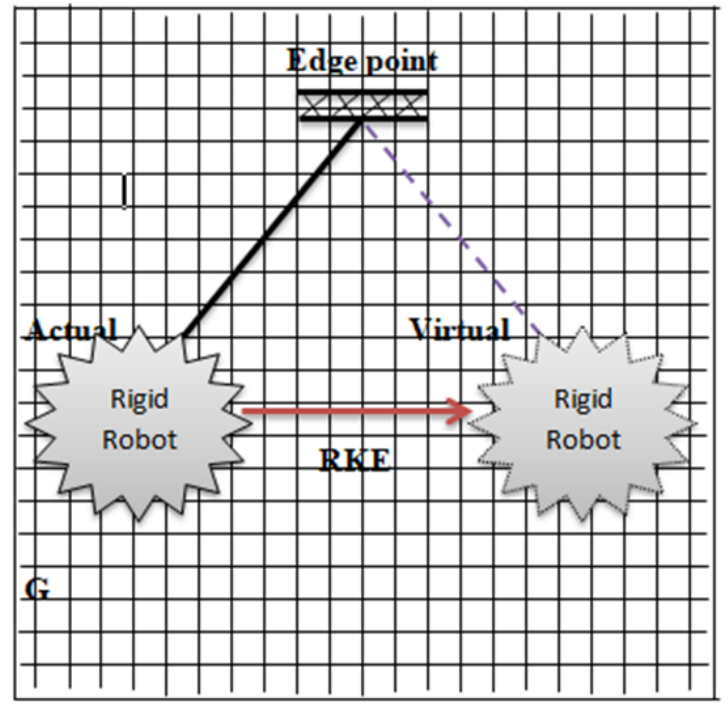

Figure 2 The sketch diagram of (RKE) algorithm placement of rigid mobile robot in a closed energy workspace.

This section highlighted the algorithm implemented on the research to drive the rigid mobile robot in other to attain rigid kinetic energy in the energy workspace.

Assuming, G represents the grid energy workspace of $\mathrm{x}$ and y coordinate, such that the rigid mobile robot is edged at a point and is deflected in a closed system.

In a closed system, the coefficient of rigid mobile robot in energy workspace is given as;

$$
\mathrm{n}=(\mathrm{KL}) /\left(\mathrm{d}^{4}\right)
$$

The deflection at the Centre of the suspended mobile robot, at the edge point, in a given energy workspace is given as;

$$
y=\left(\mathrm{KMd}^{4}\right) /\left(\mathrm{t}^{3}\right)
$$

From eq. (1), making d4 the subject of the formula, then;

$$
\mathrm{d}^{4}=(\mathrm{KL}) /(\mathrm{n})
$$

Substituting eq. (3) into eq. (2), then; 


$$
\begin{aligned}
& y=\left(K M / t^{3}\right) \times(K L / n) \\
& y=\left(K^{2} M L\right) /\left(t^{3} n\right)
\end{aligned}
$$

From eq. (4), making $M$ the subject of the formula, then;

$$
M=\left(y t^{3} n\right) /\left(K^{2} L\right)
$$

In a closed system, the rigid kinetic energy due to deflective coefficient is given as;

$$
\text { RKE }=1 / 2 \mathrm{MV}^{2}
$$

Substituting eq. (5) into eq. (6), then;

$$
R K E=1 / 2 V^{2} x\left(y t^{3} n / K^{2} L\right)
$$

Then;

$$
\mathrm{RKE}=\left(\mathrm{V}^{2} \mathrm{t}^{3} \mathrm{yn}\right) /\left(2 \mathrm{~K}^{2} \mathrm{~L}\right)
$$

Equation (8) is the rigid kinetic energy for deflective coefficient of mobile robot in energy workspace.

\subsection{The (RKE) Algorithm Flow Chart}

This section explained the step by step methodology application of the algorithm procedure for (RKE) concept.

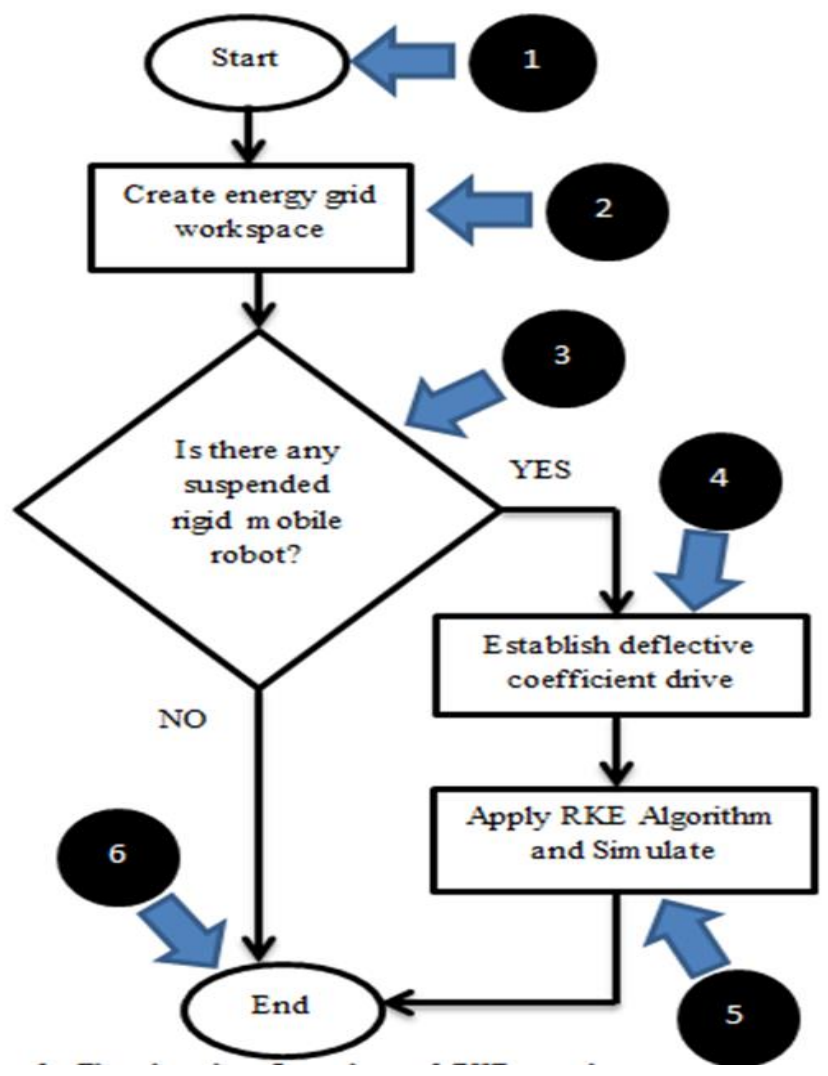

Figure 3 The algorithm flow chart of RKE step by step procedure. 


\section{Results}

This section highlighted the results obtained from the MOBOT simulation software for the rigid kinetic energy concept. In the experiment, the parameters used are stated below;

$\mathrm{t}=2.0 \mathrm{~m}, \mathrm{~V}=0.2 \mathrm{~m} / \mathrm{s}$ to $2.0 \mathrm{~m} / \mathrm{s}, \mathrm{y}=0.2 \mathrm{kgm}$ to $2.0 \mathrm{kgm}, \mathrm{n}=0.3 \mathrm{~m}-3$ to $3.0 \mathrm{~m}-3, \mathrm{~L}=0.1 \mathrm{~m}$ to $1.0 \mathrm{~m}, \mathrm{~K}=1$.

Mathematically,

$\operatorname{RKE}_{(1)}=\left[\mathrm{V}^{2}(1) \mathbf{t}^{3}(1) \mathrm{y}_{(1)} \mathrm{n}_{(1)}\right] /\left[2 \mathrm{~K}^{2} \mathrm{~L}^{(1)}\right]$

$$
\begin{aligned}
& =(0.04 \times 8 \times 0.2 \times 0.3) /(2 \times 12 \times 0.1) \\
& =(0.0192) /(0.2) \\
& =0.096 \mathrm{~J}
\end{aligned}
$$

$\operatorname{RKE}_{(2)}=\left[\mathrm{V}^{2}{ }_{(2)} \mathrm{t}^{3}{ }_{(2)} \mathrm{y}_{(2)} \mathrm{n}_{(2)}\right] /\left[2 \mathrm{~K}^{2} \mathrm{~L}_{(2)}\right]$

$$
\begin{aligned}
& =(0.16 \times 8 \times 0.4 \times 0.6) /(2 \times 12 \times 0.2) \\
& =(0.3072) /(0.4) \\
& =0.768 \mathrm{~J}
\end{aligned}
$$

$\operatorname{RKE}_{(10)}=\left[\mathrm{V}^{2}(10) \mathrm{t}^{3}{ }_{(10)} \mathrm{y}_{(10)} \mathrm{n}_{(10)}\right] /\left[2 \mathrm{~K}^{2} \mathrm{~L}_{(10)}\right]$

$$
\begin{aligned}
& =(4.00 \times 8 \times 2.0 \times 3.0) /(2 \times 12 \times 1.0) \\
& =(192) /(2.0) \\
& =96.000 \mathrm{~J}
\end{aligned}
$$

Table 1 The simulation table of Rigid Kinetic Energy (RKE) Concept in energy workspace.

\begin{tabular}{|c|c|l|l|l|}
\hline $\mathbf{S} / \mathbf{N}$ & $\mathbf{V}(\mathbf{m} / \mathbf{s})$ & $\mathbf{V}^{\mathbf{2}}(\mathbf{m} / \mathbf{s})^{\mathbf{2}}$ & $\mathbf{t}(\mathbf{m})$ & $\mathbf{t}^{\mathbf{3}}(\mathbf{m})^{\mathbf{3}}$ \\
\hline 1 & 0.2 & 0.04 & 2.0 & 8.0 \\
\hline 2 & 0.4 & 0.16 & 2.0 & 8.0 \\
\hline 3 & 0.6 & 0.36 & 2.0 & 8.0 \\
\hline 4 & 0.8 & 0.64 & 2.0 & 8.0 \\
\hline 5 & 1.0 & 1.00 & 2.0 & 8.0 \\
\hline 6 & 1.2 & 1.44 & 2.0 & 8.0 \\
\hline 7 & 1.4 & 1.96 & 2.0 & 8.0 \\
\hline 8 & 1.6 & 2.56 & 2.0 & 8.0 \\
\hline 9 & 1.8 & 3.24 & 2.0 & 8.0 \\
\hline 10 & 2.0 & 4.00 & 2.0 & 8.0 \\
\hline
\end{tabular}


Table 2 The continuous simulation table of Rigid Kinetic Energy (RKE) Concept in energy workspace

\begin{tabular}{|c|c|c|c|c|}
\hline $\mathbf{S} / \mathbf{N}$ & $\mathbf{y}(\mathbf{k g ~ m} \mathbf{)}$ & $\mathbf{n}(\mathbf{m})^{-3}$ & $\mathbf{L ~ ( m )}$ & $\mathbf{R K E ( J )}$ \\
\hline 1 & 0.2 & 0.3 & 0.1 & 0.096 \\
\hline 2 & 0.4 & 0.6 & 0.2 & 0.768 \\
\hline 3 & 0.6 & 0.9 & 0.3 & 2.592 \\
\hline 4 & 0.8 & 1.2 & 0.4 & 7.680 \\
\hline 5 & 1.0 & 1.5 & 0.5 & 12.000 \\
\hline 6 & 1.2 & 1.8 & 0.6 & 20.736 \\
\hline 7 & 1.4 & 2.1 & 0.7 & 32.928 \\
\hline 8 & 1.6 & 2.4 & 0.8 & 49.152 \\
\hline 9 & 1.8 & 2.7 & 0.9 & 69.984 \\
\hline 10 & 2.0 & 3.0 & 1.0 & 96.000 \\
\hline
\end{tabular}

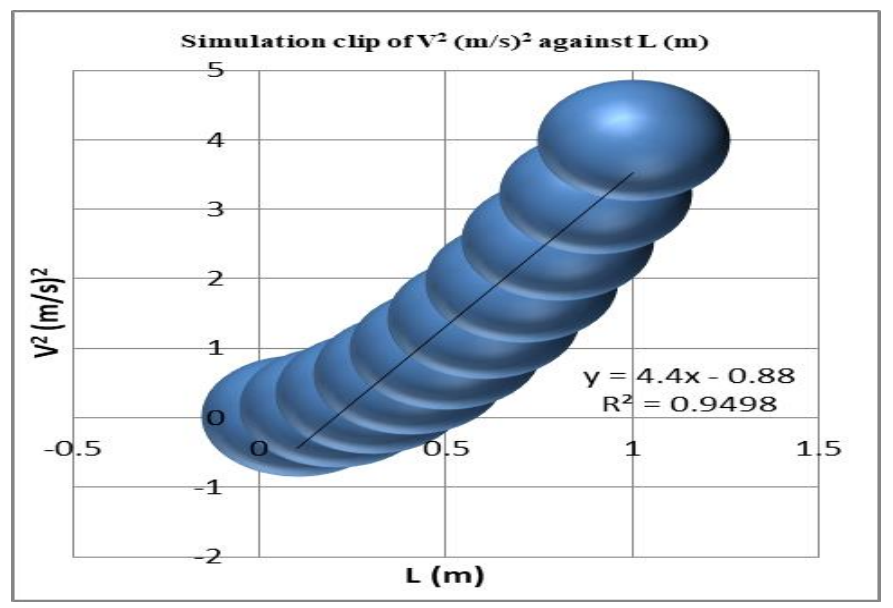

Figure 4 Simulation clip of square rigid velocity of deflective coefficient against length of deflection at the centre.

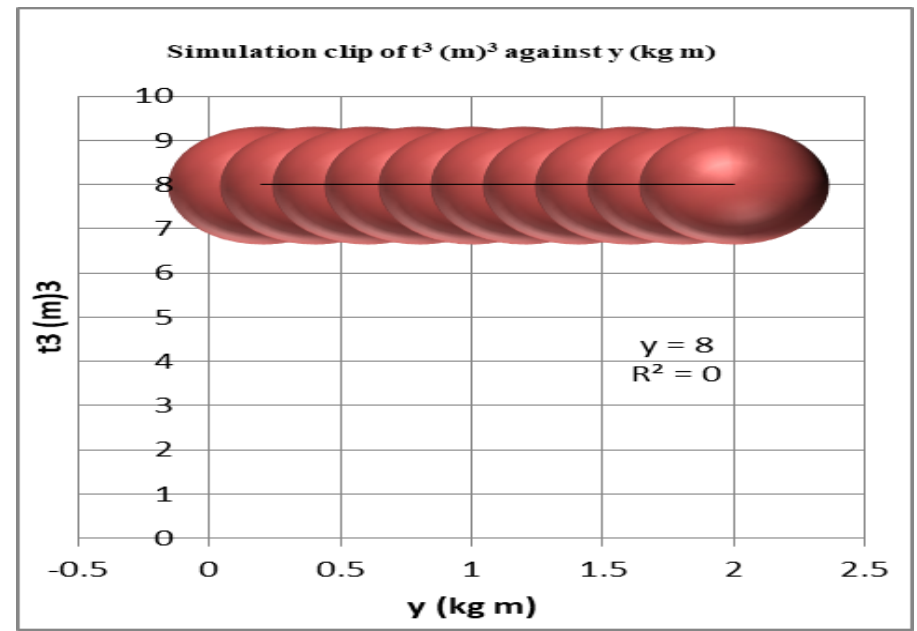

Figure 5 Simulation clip of cubic thickness of the rigid mobile robot against the deflection at the centre. 


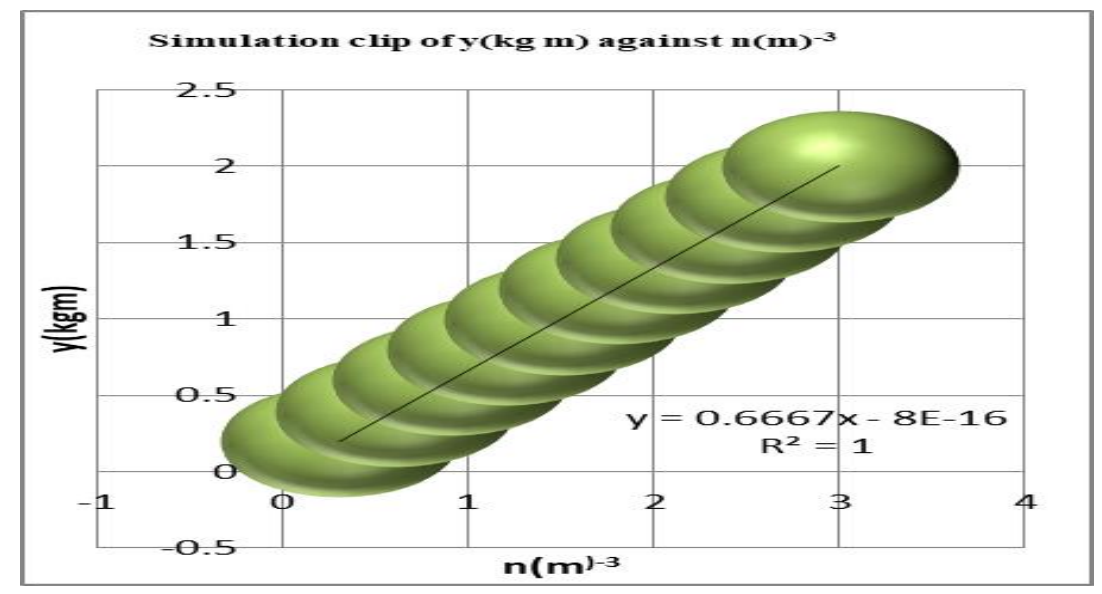

Figure 6 Simulation clip of deflection at the centre against the coefficient of rigid mobile robot.

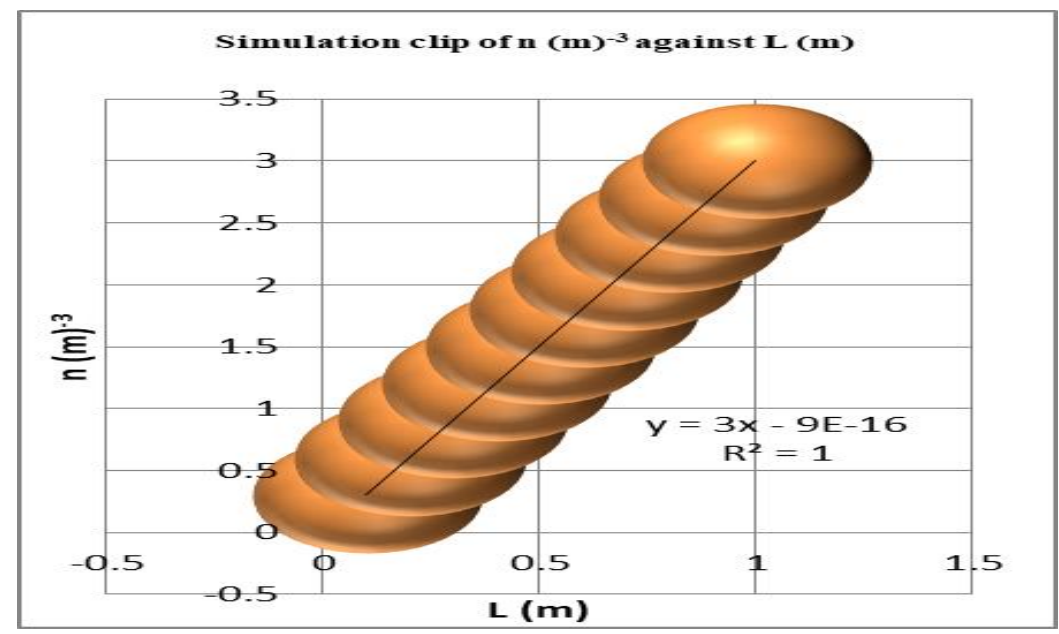

Figure 7 Simulation clip of the coefficient of rigid mobile robot against length of deflection at the centre.

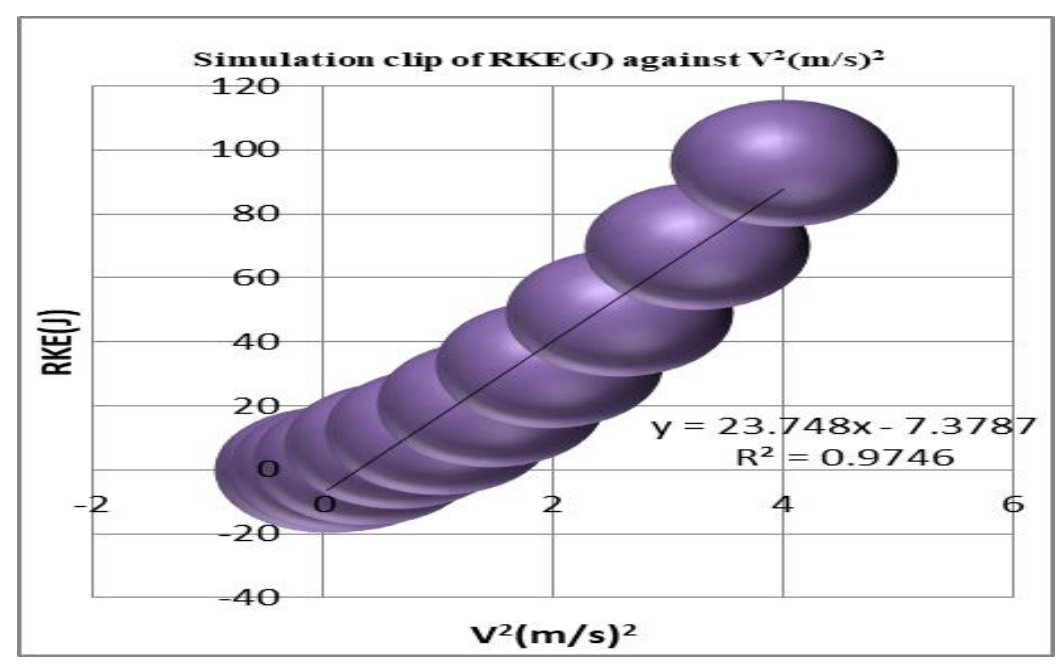

Figure 8 Simulation clip of square rigid velocity of deflective coefficient against the rigid kinetic energy of deflective coefficient of rigid mobile robot in energy workspace. 


\section{Discussion}

In figure 1, this represents the sketch diagram of rigid mobile robot on a pivoted edge point. The grey colour is the rigid mobile robot and is pivoted at a point without any energy workspace and without any deflective coefficient at the center of gravity. This is primarily the establishment of the problem statement required in other to develop an algorithm that would be test run on a robotic software application called the MOBOT software. The questions ask in the rigid kinetic energy concept on the problem statement for the experiment give rise to the robotic software parameters used in other to create a drive on the MOBOT software by the developed mathematical physics algorithm.

In figure 2, this represents the sketch diagram of the (RKE) algorithm placement of rigid mobile robot in a closed energy workspace grid. The grey rigid mobile robot is the actual robot that is deflected in other to experience kinetic energy while the dotted grey rigid mobile robot is the virtual of the actual mobile robot that undergo kinetic energy at the pivoted edge eye point in a closed energy workspace. The applications of the developed algorithm give rise to the deflective coefficient drive on the MOBOT software and established the rigid kinetic energy principle. The direction of the pointed arrow signify the deflective coefficient transformation of the rigid kinetic energy in the energy workspace of $\mathrm{x}$ and $\mathrm{y}$ coordinate from actual mobile robot state to a virtual rigid mobile robot state at the same pivoted edged eye point.

In figure 3, this represents the algorithm flow chart of (RKE) step by step methodology procedure. The flow symbols of each zone specify each stages of step by step application methodology procedure that was implemented in the research. The flow chart gives the final overview of the algorithm implementation on the MOBOT application software. It explains the stages from bullet 1 to bullet 6 , starting from start butting to the end butting.

In table 1, this represents the simulation table of Rigid Kinetic workspace is 95.004J. This is the value at which the actual mobile robot transforms to the virtual rigid mobile robot and transmits actual kinetic energy to virtual kinetic energy in the MOBOT simulation software of the closed system energy (RKE) Concept in energy workspace. In the table, lowest value of the square velocity of the deflected coefficient is $0.04 \mathrm{~m} / \mathrm{s} 2$ and the highest value of the square velocity of the deflected coefficient is $4.0 \mathrm{~m} / \mathrm{s} 2$ of the closed system in energy workspace and also in table 2, which represents the continuous simulation table of Rigid Kinetic workspace, the lowest value of the rigid kinetic energy, RKE of the deflective coefficient in energy workspace is 0.096 J and the highest value of the rigid kinetic energy, RKE of the deflective coefficient in energy workspace is 96.00J. The different between the lowest and the highest of the rigid kinetic energy, RKE of the deflective coefficient in energy workspace is 95.904J

In figure 4, this represents the simulation clip of square rigid velocity of deflective coefficient against length of deflection at the center. From the simulation clip, the blue object is the cascaded rigid mobile robot under the influence of rigid kinetic energy algorithm in the MOBOT simulation software, also exhibiting velocity of deflective coefficient depending on the thickness of the mobile robot and the influence of length of deflection at the center of gravity in energy workspace. The virtual rigid kinetic energy obtained is as a result of actual mobile robot transformation to virtual rigid mobile robot which generate a longitudinal equation of $\mathrm{y}=4.4 \mathrm{x}-0.88$ in the closed system, and also a linear gradient of $\mathrm{R} 2=0.9498$ in the closed system of the energy workspace. At each cascade of the rigid mobile robot, is as a result of the ten consecutives time the deflection coefficient made a virtual rigid mobile robot transformation from the actual mobile robot and the actual kinetic energy is transmitted to the virtual kinetic energy in the closed system of the energy workspace.

In figure 5, this represents the simulation clip of cubic thickness of the rigid mobile robot against the deflection at the center. From the simulation clip, the brown object is the cascaded rigid mobile robot under the influence of rigid kinetic energy algorithm in the MOBOT simulation software, also exhibiting square rigid velocity of deflective coefficient depending on the cubic thickness of the rigid mobile robot and the influence of deflection at the center of gravity in energy workspace. The virtual rigid kinetic energy obtained is as a result of actual mobile robot transformation to virtual rigid mobile robot which generate a longitudinal equation of $\mathrm{y}=8$ in the closed system, and also a linear gradient of $\mathrm{R} 2$ $=0$ in the closed system of the energy workspace. At each cascade of the rigid mobile robot, is as a result of the ten consecutives time the deflection coefficient made a virtual rigid mobile robot transformation from the actual mobile robot and the actual kinetic energy is transmitted to the virtual kinetic energy in the closed system of the energy workspace.

In figure 6, this represents the simulation clip of deflection at the center against the coefficient of rigid mobile robot. From the simulation clip, the green object is the cascaded rigid mobile robot under the influence of rigid kinetic energy algorithm in the MOBOT simulation software, also exhibiting coefficient of rigid mobile robot depending on the thickness of the mobile robot and the influence of deflection at the center of gravity with square rigid velocity of 
deflection coefficient in energy workspace. The virtual rigid kinetic energy obtained is as a result of actual mobile robot transformation to virtual rigid mobile robot which generate a longitudinal equation of $y=0.6667 \mathrm{x}-8 \mathrm{E}-16$ in the closed system, and also a linear gradient of R2 = 1 in the closed system of the energy workspace. At each cascade of the rigid mobile robot, is as a result of the ten consecutives time the deflection coefficient made a virtual rigid mobile robot transformation from the actual mobile robot and the actual kinetic energy is transmitted to the virtual kinetic energy in the closed system of the energy workspace.

In figure 7, this represents the simulation clip of the coefficient of rigid mobile robot against length of deflection at the center. From the simulation clip, the orange object is the cascaded rigid mobile robot under the influence of rigid kinetic energy algorithm in the MOBOT simulation software, also exhibiting coefficient of rigid mobile robot depending on the thickness of the mobile robot and the influence of length of deflection at the center of gravity with square rigid velocity of deflection coefficient in energy workspace. The virtual rigid kinetic energy obtained is as a result of actual mobile robot transformation to virtual rigid mobile robot which generate a longitudinal equation of $y=3 \mathrm{x}-9 \mathrm{E}-16$ in the closed system, and also a linear gradient of R2 = 1 in the closed system of the energy workspace. At each cascade of the rigid mobile robot, is as a result of the ten consecutives time the deflection coefficient made a virtual rigid mobile robot transformation from the actual mobile robot and the actual kinetic energy is transmitted to the virtual kinetic energy in the closed system of the energy workspace.

In figure 8, this represents the simulation clip of square rigid velocity of deflective coefficient against the rigid kinetic energy for deflective coefficient of rigid mobile robot in energy workspace. From the simulation clip, the pink object is the cascaded rigid mobile robot under the influence of rigid kinetic energy algorithm in the MOBOT simulation software, also exhibiting coefficient of rigid mobile robot depending on the thickness of the mobile robot and the influence of deflection at the center of gravity with square rigid velocity of deflection coefficient in energy workspace. The virtual rigid kinetic energy obtained is as a result of actual mobile robot transformation to virtual rigid mobile robot which generate a longitudinal equation of $\mathrm{y}=23.748 \mathrm{x}-7.3787$ in the closed system, and also a linear gradient of $\mathrm{R} 2=0.9746$ in the closed system of the energy workspace. At each cascade of the rigid mobile robot, is as a result of the ten consecutives time the deflection coefficient made a virtual rigid mobile robot transformation from the actual mobile robot and the actual kinetic energy is transmitted to the virtual kinetic energy in the closed system of the energy workspace.

\section{Conclusion}

This concept has practically proved the originality of rigid kinetic energy for deflective suspended mobile robot in energy workspace, in a partially known environment (PKE). The simulation of the algorithm, used to obtain the results has proved to be robust, efficient, $100 \%$ effective and valid. The simulated clip obtained from (RKE) concept analysis is highly classified and represents the true nature of the algorithm. The limitation encountered during the research includes lack of personal funding encountered, in which i have to work extra man hours in the industry where is currently working with as data engineer, the challenge of sourcing for MOBOT simulation software and books relating to the problem statements. In the future, am looking forward in expanding my scope in meeting NGOs to raise fund and PhD working scholarship to make more research and finally engage in future research papers titled; rigid potential energy concept for autonomous mobile robot navigation and the suspended velocity motion for unmanned mobile robot path planning.

\section{Compliance with ethical standards}

\section{Acknowledgments}

I acknowledge the God Almighty for the grace he gave me to carry out this research and the good will of my wife Mrs. Stella Akwara for the endurance she has over me during the research process.

\section{Disclosure of conflict of interest}

The author of this research has no corresponding author attached to this research. 


\section{References}

[1] Maxwell JC. (1890). Rigid Body Dynamics: Rotation \& Translation about a Fixed Axis. Niven Journal Edition, 1(248), 1-29.

[2] Pandey NC. (2020). Revolutionary Motion of a Body under the Action of Resolution of Acceleration due to gravity involved, International Journal of Scientific Research in Physics and Applied Sciences, 8(4), 27-34.

[3] Guendelman et al. (2003). Non-convex Rigid Bodies with Stacking, ACM Transactions on Graphics Journal, 22(3), 871-878.

[4] Dingle B. (2004). Rigid Body Motion (An Introduction), Texas A \& M University, 1-23.

[5] Ni M. (2014). Study on the Application of Rigid Body Dynamics in the Traffic Accident Reconstruction, Applied Mechanics \& Materials, Trans Tech Publications, Ltd, 707, 412-416.

[6] Popovic et al. (2000). Interactive Manipulation of Rigid Body Simulation, Proceedings of SIGGRAPH, 209-218.

[7] Semat et al. (1958). Physics, Chapter 11: Rotational Motion (The Dynamics of Rigid Body), Research paper in Physics \& Astronomy, 141, 198-224.

[8] Bhat et al. (2002). Computing the Physical Parameters of Rigid-Body Motion from Video, Proceeding of bhateccv02, 1-16.

[9] Peraire et al. (2008). 3D Rigid Body Dynamics: Kinetic Energy; Instability; Equations of Motion, 16.07 Dynamic, Lecture L27, Version 2.0, 1-11.

[10] Hurtado et al. (2004) Hamel Coefficients for the Rotational Motion of an N-Dimensional Rigid Body, Proceedings of the Royal Society, A Mathematical Physical \& Engineering Sciences, 460(2052), 3613-3630.

[11] Chang et al. (2016). The Design \& Experiments of a Small Wheel-Legged Mobile Robot System with two Robotic Arms, IEEE/RSJ International Conference on Intelligent Robots and Systems (IROS), Daejeon, 2590-2595.

[12] Stroud et al. (2001). Engineering Mathematics, Programmed 10, Partial Differentiation 1, Fifth edition, England, 690.

[13] Koshiyama et al. (1993). Design \& Control of an All-Direction Steering type Mobile Robot, The International Journal of Robotic Research, 12(5), 411-419.

[14] Maurette M. (2003). Mars Rover Autonomous Navigation, Autonomous Robot, 14 (2), 199-208.

[15] Billah et al. (2008). Walking Hexapod Robot in Disaster Recovery: Developing Algorithm for Terrain Negotiation \& Navigation, World Academy of Science, Engineering and Technology, International Journal of Mechanical and Mechatronics Engineering, 2(4), 795-800. 